\author{
Mykola IVAnChov, Vitaliy Vlasov
}

\title{
INVERSE PROBLEM FOR A 2D STRONGLY DEGENERATE HEAT EQUATION WITH INTEGRAL OVERDETERMINATION CONDITIONS
}

\footnotetext{
The existence of a classical solution to the problem of identification of two time-dependent coefficients in the $2 \mathrm{D}$ heat equation is considered. We suppose that unknown coefficients vanish at the initial moment of time as a power with exponent greater than 1. Existence is established via Schauder fixed-point theorem.

Key words and phrases: Inverse problem, 2D strongly degenerate heat equation, integral overdetermination conditions.
}

\begin{abstract}
Ivan Franko National University of Lviv, Lviv, Ukraine (Mykola Ivanchov)
Ivan Franko National University of Lviv, Lviv, Ukraine (Vitaliy Vlasov)

e-mail: mykola.ivanchov@lnu.edu.ua (Mykola Ivanchov), siphiuel@gmail.com (Vitaliy Vlasov)
\end{abstract}

\section{INTRODUCTION}

An inverse problem for a 2D strongly degenerate anisotropic heat equation in a rectangular domain is considered. Such problems are of growing importance to various branches of physics, economics, etc. [1, 2, 3]. The first results on identification of unknown coefficients in degenerate equations were obtained in $[4,5]$. More systematic investigation of inverse problems for one-dimensional degenerate parabolic equations was made in $[6,7,8]$. The transition to $2 \mathrm{D}$ degenerate equations was realized only in the case when unknown coefficients depend on the time variable. For weakly degenerate parabolic equations, inverse problems with one or two unknown leading coefficients $[9,10]$ were studied. An inverse problem for 2D strongly degenerate heat equation with one unknown coefficient was investigated in [11].

In this paper we establish conditions for existence of solution to an inverse problem for a 2D strongly degenerate heat equation with two unknown coefficients that have different behavior of power type at the initial moment of time.

\section{Problem STATEMENT}

In the domain $Q_{T}:=\{(x, y, t): 0<x<h, 0<y<l, 0<t<T\}$ we consider a two-dimensional heat equation with two unknown leading coefficients depending on the time

УДК 517.95

2010 Mathematics Subject Classification: 35R30, 35K65.

(C) Mykola Ivanchov, Vitaliy Vlasov, 2019 
variable. We suppose that each of unknown coefficients vanishes at the initial moment of time as a power $t^{\beta_{i}}$ where $\beta_{i} \geq 1, i \in\{1,2\}$. We will use mixed Dirichlet-Neumann boundary conditions, and the additional conditions (also called overdetermination conditions) will be of integral type. The problem is to find a triplet of functions $\left(a_{1}(t), a_{2}(t), u(x, y, t)\right), a_{i}(t)>$ $0, t \in(0, T], i \in\{1,2\}$ satisfying the degenerate heat equation

$$
u_{t}=a_{1}(t) u_{x x}+a_{2}(t) u_{y y}+f(x, y, t), \quad(x, y, t) \in Q_{T},
$$

initial condition

$$
u(x, y, 0)=\varphi(x, y, 0), \quad(x, y) \in \bar{D}:=[0, h] \times[0, l],
$$

boundary conditions

$$
\begin{aligned}
& u(0, y, t)=\mu_{11}(y, t), u(h, y, t)=\mu_{12}(y, t), \quad(y, t) \in[0, l] \times[0, T], \\
& u_{y}(x, 0, t)=\mu_{21}(x, t), u_{y}(x, l, t)=\mu_{22}(x, t), \quad(x, t) \in[0, h] \times[0, T]
\end{aligned}
$$

and overdetermination conditions

$$
\iint_{D} u(x, y, t) d x d y=\mu_{31}(t), \quad \iint_{D} x u(x, y, t) d x d y=\mu_{32}(t), \quad t \in(0, T] .
$$

Our aim is to determine the conditions of existence of classical solution to the problem (1)-(5).

\section{Reduction of the problem (1)-(5) TO An EQuAtion System For $a_{1}(t), a_{2}(t)$}

Assume temporarily that coefficients $a_{i}=a_{i}(t)>0, t \in(0, T]$ from the space $C[0, T]$ are known. Reduce the problem (1)-(5) to a system of equations with respect to $a_{1}(t), a_{2}(t)$ with the aid of overdetermination conditions (5). Differentiating them with respect to $t$ and using equation (1) we obtain

$$
\begin{aligned}
& a_{1}(t) \int_{0}^{l}\left(h u_{x}(h, y, t)+\mu_{11}(y, t)-\mu_{12}(y, t)\right) d y+a_{2}(t) \int_{0}^{h} x\left(\mu_{22}(x, t)-\mu_{21}(x, t)\right) d x \\
& =\mu_{32}^{\prime}(t)-\iint_{D} x f(x, y, t) d x d y, \quad t \in[0, T] \\
& a_{1}(t) \int_{0}^{l}\left(u_{x}(h, y, t)-u_{x}(0, y, t)\right) d y+a_{2}(t) \int_{0}^{h}\left(\mu_{22}(x, t)-\mu_{21}(x, t)\right) d x \\
& =\mu_{31}^{\prime}(t)-\iint_{D} f(x, y, t) d x d y, \quad t \in[0, T]
\end{aligned}
$$


Transform the system of equations (6), (7), substituting the values related to $u$. Using the Green function $G_{12}(x, y, t, \xi, \eta, \tau)$ we find the solution to the direct problem (1)-(4)

$$
\begin{aligned}
& u(x, y, t)=\int_{0}^{l} \int_{0}^{h} G_{12}(x, y, t, \xi, \eta, 0) \varphi(\xi, \eta) d \xi d \eta+\int_{0}^{t} \int_{0}^{l} G_{12_{\xi}}(x, y, t, 0, \eta, \tau) a_{1}(\tau) \\
& \times \mu_{1}(\eta, \tau) d \eta d \tau-\int_{0}^{t} \int_{0}^{l} G_{12_{\xi}}(x, y, t, h, \eta, \tau) a_{1}(\tau) \mu_{2}(\eta, \tau) d \eta d \tau-\int_{0}^{t} \int_{0}^{h} G_{12}(x, y, t, \xi, 0, \tau) \\
& \times a_{2}(\tau) \nu_{1}(\xi, \tau) d \xi d \tau+\int_{0}^{t} \int_{0}^{h} G_{12}(x, y, t, \xi, l, \tau) a_{2}(\tau) \nu_{2}(\xi, \tau) d \xi d \tau \\
& +\int_{0}^{t} \int_{D} G_{12}(x, y, t, \xi, \eta, \tau) f(\xi, \eta, \tau) d \xi d \eta d \tau, \quad(x, y, t) \in \bar{Q}_{T} .
\end{aligned}
$$

The Green function is defined by the equality

$$
\begin{aligned}
& G_{i j}(x, y, t, \xi, \eta, \tau)=\frac{1}{4 \pi \sqrt{\left(\theta_{1}(t)-\theta_{1}(\tau)\right)\left(\theta_{2}(t)-\theta_{2}(\tau)\right)}} \sum_{m, n=-\infty}^{\infty}\left(\exp \left(-\frac{(x-\xi+2 n h)^{2}}{4\left(\theta_{1}(t)-\theta_{1}(\tau)\right)}\right)\right. \\
& \left.+(-1)^{i} \exp \left(-\frac{(x+\xi+2 n h)^{2}}{4\left(\theta_{1}(t)-\theta_{1}(\tau)\right)}\right)\right)\left(\exp \left(-\frac{(y-\eta+2 m l)^{2}}{4\left(\theta_{2}(t)-\theta 21(\tau)\right)}\right)\right) \\
& \left.+(-1)^{j} \exp \left(-\frac{(y+\eta+2 m l)^{2}}{4\left(\theta_{2}(t)-\theta_{2}(\tau)\right)}\right)\right), \quad i, j \in\{1,2\}, \theta_{k}(t):=\int_{0}^{t} a_{k}(\sigma) d \sigma, k \in\{1,2\},
\end{aligned}
$$

where the values $i, j=1$ correspond to Dirichlet boundary conditions and $i, j=2$ to Neumann conditions by $x$ and $y$ respectively. One can see that $2 \mathrm{D}$ Green function $G_{i j}$ may be presented as a product of two 1D Green functions for corresponding heat equations: $G_{i j}(x, y, t, \xi, \eta, \tau)=G_{i}(x, t, \xi, \tau) G_{j}(y, t, \eta, \tau)$. Moreover, it is easy to verify that $G_{1_{x}}(x, t, \xi, \tau)=-G_{2_{\xi}}(x, t, \xi, \tau)$.

Suppose that the following assumptions hold:

(A1) $\varphi \in C^{1,0}(\bar{D}), \mu_{1 i} \in C^{2,1}([0, l] \times(0, T]), \mu_{2 i} \in C^{1,0}([0, h] \times(0, T]), f \in C^{1,0,0}\left(\bar{Q}_{T}\right), \mu_{3 i} \in$ $C^{1}([0, T]), i \in\{1,2\}$.

Here we used the notations from [12]. Now, find the derivative $u_{x}(x, y, t)$ and transform 
it by integrating by parts:

$$
\begin{aligned}
& u_{x}(x, y, t)=\int_{0}^{l} \int_{0}^{h} G_{22}(x, y, t, \xi, \eta, 0) \varphi_{\xi}(\xi, \eta) d \xi d \eta \\
& -\int_{0}^{t} \int_{0}^{l} G_{22}(x, y, t, 0, \eta, \tau)\left(\mu_{11_{\tau}}(\eta, \tau)-a_{2}(\tau) \mu_{11_{\eta \eta}}(\eta, \tau)-f(0, \eta, \tau)\right) d \eta d \tau \\
& +\int_{0}^{t} \int_{0}^{l} G_{22}(x, y, t, h, \eta, \tau)\left(\mu_{12_{\tau}}(\eta, \tau)-a_{2}(\tau) \mu_{12_{\eta \eta}}(\eta, \tau)-f(h, \eta, \tau)\right) d \eta d \tau \\
& -\int_{0}^{t} \int_{0}^{h} G_{22}(x, y, t, \xi, 0, \tau) a_{2}(\tau) \mu_{21_{\xi}}(\xi, \tau) d \xi d \tau+\int_{0}^{t} \int_{0}^{h} G_{22}(x, y, t, \xi, l, \tau) \\
& \times a_{2}(\tau) \mu_{22_{\xi}}(\xi, \tau) d \xi d \tau+\int_{0}^{t} \iint_{D} G_{22}(x, y, t, \xi, \eta, \tau) f_{\xi}(\xi, \eta, \tau) d \xi d \eta d \tau .
\end{aligned}
$$

To find $\int_{0}^{l} u_{x}(x, y, t) d y$, note that by direct calculation we get $\int_{0}^{h} G_{2}(x, t, \xi, \tau) d x=1$. Hence, we obtain

$$
\begin{aligned}
& \int_{0}^{l} u_{x}(x, y, t) d y=\int_{0}^{h} G_{2}(x, t, \xi, 0) d \xi \int_{0}^{l} \varphi_{\xi}(\xi, \eta) d \eta-\int_{0}^{t} G_{2}(x, t, 0, \tau) d \tau \int_{0}^{l}\left(\mu_{11_{\tau}}(\eta, \tau)\right. \\
& -f(0, \eta, \tau)) d \eta+\int_{0}^{t} G_{2}(x, t, h, \tau) d \tau \int_{0}^{l}\left(\mu_{12_{\tau}}(\eta, \tau)-f(h, \eta, \tau)\right) d \eta \\
& +\int_{0}^{t} G_{2}(x, t, 0, \tau) a_{2}(\tau)\left(\mu_{22}(0, \tau)-\mu_{21}(0, \tau)\right) d \tau-\int_{0}^{t} G_{2}(x, t, h, \tau) a_{2}(\tau)\left(\mu_{22}(h, \tau)\right. \\
& \left.-\mu_{21}(h, \tau)\right) d \tau-\int_{0}^{t} \int_{0}^{h} G_{2}(x, t, \xi, \tau) a_{2}(\tau) \mu_{21_{\xi}}(\xi, \tau) d \xi d \tau+\int_{0}^{t} \int_{0}^{h} G_{2}(x, t, \xi, \tau) \\
& \times a_{2}(\tau) \mu_{22_{\xi}}(\xi, \tau) d \xi d \tau+\int_{0}^{t} \int_{0}^{h} G_{2}(x, t, \xi, \tau) d \xi d \tau \int_{0}^{l} f_{\xi}(\xi, \eta, \tau) d \eta .
\end{aligned}
$$

Return to the system $(6),(7)$. To find its positive solution on $(0, T]$, consider an auxiliary algebraic system of equations

$$
a_{1} x+b_{1} y=c_{1}, \quad a_{2} x+b_{2} y=c_{2}
$$

where $a_{i}>0, b_{i}>0, c_{i}>0, i \in\{1,2\}$. The solution of this system is defined by a formula

$$
x=\frac{b_{2} c_{1}-b_{1} c_{2}}{a_{1} b_{2}-a_{2} b_{1}}, \quad y=\frac{a_{1} c_{2}-a_{2} c_{1}}{a_{1} b_{2}-a_{2} b_{1}} .
$$


It has a positive solution if

$$
b_{2} c_{1}-b_{1} c_{2}>0, \quad a_{1} c_{2}-a_{2} c_{1}>0, \quad a_{1} b_{2}-a_{2} b_{1}>0 .
$$

In turn, these conditions are fulfilled if

$$
\frac{a_{1}}{a_{2}}>\frac{c_{1}}{c_{2}}>\frac{b_{1}}{b_{2}}
$$

Apply this result to the system (6), (7). It follows that the positive solution of the system (6), (7) exists if the following conditions hold:

$$
\begin{aligned}
& \mu_{22}(x, t)-\mu_{21}(x, t) \geq 0, \mu_{22}(x, t) \not \equiv \mu_{21}(x, t),(x, t) \in[0, h] \times(0, T], \quad \int_{0}^{l}\left(\mu_{11}(\eta, t)\right. \\
& \left.-\mu_{12}(\eta, t)\right) d \eta>0, \quad \mu_{31}^{\prime}(t)-\iint_{D} f(x, y, t) d x d y>0, \quad \mu_{32}^{\prime}(t)-\iint_{D} x f(x, y, t) d x d y>0, \\
& h>\frac{\mu_{32}^{\prime}(t)-\iint_{D} x f(x, y, t) d x d y, \quad \int_{D}^{h} x\left(\mu_{22}(x, t)-\mu_{21}(x, t)\right) d x}{\mu_{31}^{\prime}(t)-\iint_{0} f(x, y, t) d x d y}>\frac{\int_{0}^{h}\left(\mu_{22}(x, t)-\mu_{21}(x, t)\right) d x}{\int_{0}^{l} u_{x}(0, y, t) d y \geq 0, \quad \int_{0}^{l}\left(u_{x}(h, y, t)-u_{x}(0, y, t)\right) d y>0, \quad t \in(0, T] .}
\end{aligned}
$$

Note that two last conditions in (12) are expressed via $u(x, y, t)$. In order to study the first of them, put $x=0$ in (10) and consider one of the terms within:

$$
\begin{aligned}
& \int_{0}^{t} G_{2}(0, t, 0, \tau) a_{2}(\tau)\left(\mu_{22}(0, \tau)-\mu_{21}(0, \tau)\right) d \tau-\int_{0}^{t} G_{2}(0, t, h, \tau) a_{2}(\tau)\left(\mu_{22}(h, \tau)\right. \\
& \left.-\mu_{21}(h, \tau)\right) d \tau=\int_{0}^{t}\left(G_{2}(0, t, 0, \tau)-G_{2}(0, t, h, \tau)\right) a_{2}(\tau)\left(\mu_{22}(0, \tau)-\mu_{21}(0, \tau)\right) d \tau \\
& +\int_{0}^{t} G_{2}(0, t, h, \tau) a_{2}(\tau)\left(\mu_{22}(0, \tau)-\mu_{21}(0, \tau)-\mu_{22}(h, \tau)+\mu_{21}(h, \tau)\right) d \tau
\end{aligned}
$$

As $G_{2}(0, t, 0, \tau)-G_{2}(0, t, h, \tau) \geq 0$, we have $\int_{0}^{l} u_{x}(0, y, t) d y>0$ if the following assumptions are fulfilled:

$$
\begin{aligned}
& \int_{0}^{l}\left(\mu_{11_{t}}(y, t)-f(0, y, t)\right) d y<0, \int_{0}^{l}\left(\mu_{12_{t}}(y, t)-f(h, y, t)\right) d y>0, t \in[0, T], \int_{0}^{l} \varphi_{x}(x, y) d y>0, \\
& \mu_{21_{x}}(x, t) \leq 0, \mu_{22_{x}}(x, t) \geq 0, \mu_{22}(x, t)-\mu_{21}(x, t) \geq 0, \mu_{22}(x, t) \not \equiv \mu_{21}(x, t), \int_{0}^{l} f_{x}(x, y, t) d y \\
& \geq 0,(x, t) \in[0, h] \times(0, T], \mu_{22}(0, t)-\mu_{21}(0, t)-\mu_{22}(h, t)+\mu_{21}(h, t) \geq 0 .
\end{aligned}
$$


Find from (10)

$$
\begin{aligned}
& \int_{0}^{l}\left(u_{x}(h, y, t)-u_{x}(0, y, t)\right) d y=\int_{0}^{h}\left(G_{2}(h, t, \xi, 0)-G_{2}(0, t, \xi, 0) d \xi \int_{0}^{l} \varphi_{\xi}(\xi, \eta) d \eta\right. \\
& -\int_{0}^{t}\left(G_{2}(h, t, 0, \tau)-G_{2}(0, t, 0, \tau)\right) d \tau \int_{0}^{l}\left(\mu_{11_{\tau}}(\eta, \tau)-f(0, \eta, \tau)+\mu_{12_{\tau}}(\eta, \tau)-f(h, \eta, \tau)\right) d \eta \\
& +\int_{0}^{t}\left(G_{2}(h, t, 0, \tau)-G_{2}(0, t, 0, \tau)\right) a_{2}(\tau)\left(\mu_{22}(0, \tau)-\mu_{21}(0, \tau)+\mu_{22}(h, \tau)-\mu_{21}(h, \tau)\right) d \tau \\
& +\int_{0}^{t} \int_{0}^{h}\left(G_{2}(h, t, \xi, \tau)-G_{2}(0, t, \xi, \tau)\right) a_{2}(\tau)\left(\mu_{22_{\xi}}(\xi, \tau)-\mu_{21_{\xi}}(\xi, \tau)\right) d \xi d \tau \\
& +\int_{0}^{t} \int_{0}^{h}\left(G_{2}(h, t, \xi, \tau)-G_{2}(0, t, \xi, \tau)\right) d \xi d \tau \int_{0}^{l} f_{\xi}(\xi, \eta, \tau) d \eta .
\end{aligned}
$$

Taking into account the representation of the Green function, consider

$$
\begin{aligned}
& G_{2}(0, t, \xi, \tau)-G_{2}(h, t, \xi, \tau)=\frac{1}{\sqrt{\pi\left(\theta_{1}(t)-\theta_{1}(\tau)\right)}} \sum_{n=-\infty}^{\infty}\left(\exp \left(-\frac{(\xi+2 n h)^{2}}{4\left(\theta_{1}(t)-\theta_{1}(\tau)\right)}\right)\right. \\
& \left.-\exp \left(-\frac{(\xi+(2 n+1) h)^{2}}{4\left(\theta_{1}(t)-\theta_{1}(\tau)\right)}\right)\right)=\frac{1}{\sqrt{\pi\left(\theta_{1}(t)-\theta_{1}(\tau)\right)}} \sum_{n=-\infty}^{\infty}(-1)^{n} \exp \left(-\frac{(\xi+n h)^{2}}{4\left(\theta_{1}(t)-\theta_{1}(\tau)\right)}\right) \\
& :=\tilde{G}(0, t, \xi, \tau) .
\end{aligned}
$$

It is easy to check that $\tilde{G}(x, t, \xi, \tau)$ is the Green function for the heat equation

$$
u_{t}=a_{1}(t) u_{x x}, \quad(x, t) \in(0, h / 2) \times(0, T)
$$

with boundary conditions

$$
\tilde{G}_{x}(0, t, \xi, \tau)=0, \quad \tilde{G}(h / 2, t, \xi, \tau)=0 .
$$

In virtue of properties of Green functions, $\tilde{G}(x, t, \xi, \tau) \geq 0,(x, t),(\xi, \tau) \in[0, h / 2] \times[0, T]$.

For some continuous on $[0, h]$ function $\psi$, transform the integral

$$
\begin{aligned}
& \int_{0}^{h}\left(G_{2}(h, t, \xi, \tau)-G_{2}(0, t, \xi, \tau)\right) \psi(\xi) d \xi=\frac{1}{\sqrt{\pi\left(\theta_{1}(t)-\theta_{1}(\tau)\right)}} \int_{0}^{h} \sum_{n=-\infty}^{\infty}(-1)^{n+1} \\
& \times \exp \left(-\frac{(\xi+n h)^{2}}{4\left(\theta_{1}(t)-\theta_{1}(\tau)\right)}\right) \psi(\xi) d \xi=\frac{1}{\sqrt{\pi\left(\theta_{1}(t)-\theta_{1}(\tau)\right)}} \int_{0}^{h / 2} \sum_{n=-\infty}^{\infty}(-1)^{n+1} \\
& \times \exp \left(-\frac{(\xi+n h)^{2}}{4\left(\theta_{1}(t)-\theta_{1}(\tau)\right)}\right) \psi(\xi) d \xi+\frac{1}{\sqrt{\pi\left(\theta_{1}(t)-\theta_{1}(\tau)\right)}} \int_{h / 2}^{h} \sum_{n=-\infty}^{\infty}(-1)^{n+1} \\
& \times \exp \left(-\frac{(\xi+n h)^{2}}{4\left(\theta_{1}(t)-\theta_{1}(\tau)\right)}\right) \psi(\xi) d \xi .
\end{aligned}
$$


After substitution $\zeta=h-\xi$ in the second integral, we obtain

$$
\int_{0}^{h}\left(G_{2}(h, t, \xi, \tau)-G_{2}(0, t, \xi, \tau)\right) \psi(\xi) d \xi=\int_{0}^{h / 2} \tilde{G}(0, t, \xi, \tau)(\psi(h-\xi)-\psi(\xi)) d \xi .
$$

The same transformation is applied also to the last integral in (14). Hence, the expression (14) is reduced to the form

$$
\begin{aligned}
& \int_{0}^{l}\left(u_{x}(h, y, t)-u_{x}(0, y, t)\right) d y=\int_{0}^{h / 2} \tilde{G}(0, t, \xi, \tau) d \xi \int_{0}^{l}\left(\varphi_{\xi}(h-\xi, \eta)-\varphi_{\xi}(\xi, \eta)\right) d \eta \\
& +\int_{0}^{t} \tilde{G}(0, t, 0, \tau) d \tau \int_{0}^{l}\left(\mu_{11_{\tau}}(\eta, \tau)-f(0, \eta, \tau)+\mu_{12_{\tau}}(\eta, \tau)-f(h, \eta, \tau)\right) d \eta \\
& -\int_{0}^{t} \tilde{G}(0, t, \xi, \tau) a_{2}(\tau)\left(\mu_{22}(0, \tau)-\mu_{21}(0, \tau)+\mu_{22}(h, \tau)-\mu_{21}(h, \tau)\right) d \tau \\
& +\int_{0}^{t} \int_{0}^{h / 2} \tilde{G}(0, t, \xi, \tau) a_{2}(\tau)\left(\mu_{22_{\xi}}(h-\xi, \tau)-\mu_{21_{\xi}}(h-\xi, \tau)-\mu_{22_{\xi}}(\xi, \tau)+\mu_{21_{\xi}}(\xi, \tau)\right) d \xi d \tau \\
& +\int_{0}^{t} \int_{0}^{h / 2} \tilde{G}(0, t, \xi, \tau) d \xi d \tau \int_{0}^{l}\left(f_{\xi}(h-\xi, \eta, \tau)-f_{\xi}(\xi, \eta, \tau)\right) d \eta
\end{aligned}
$$

Taking into account (12)-(15), we conclude that the system (6), (7) has a positive solution 
if the following assumptions hold:

(A2) $\int_{0}^{l} \varphi_{x}(x, y) d y>0, \mu_{21_{x}}(x, t) \leq 0, \mu_{22_{x}}(x, t) \geq 0, \int_{0}^{l} f_{x}(x, y, t) d y \geq 0, \mu_{22}(x, t) \not \equiv \mu_{21}(x, t)$,
$\mu_{22}(x, t)-\mu_{21}(x, t) \geq 0, \int_{0}^{l}\left(\varphi_{x}(h-x, \eta)-\varphi_{x}(x, \eta)\right) d \eta \geq 0, \int_{0}^{l}\left(\mu_{11_{t}}(\eta, t)-f(0, \eta, t)\right.$
$\left.+\mu_{12_{t}}(\eta, t)-f(h, \eta, t)\right) d \eta \geq 0, \mu_{22_{x}}(h-x, t)-\mu_{21_{x}}(h-x, t)-\mu_{22_{x}}(x, t)+\mu_{21_{x}}(x, t) \geq 0$
$\int_{0}^{l}\left(f_{x}(h-x, \eta, t)-f_{x}(x, \eta, t)\right) d \eta \geq 0,(x, t) \in[0, h] \times(0, T], \int_{0}^{l}\left(\mu_{11}(y, t)-\mu_{12}(y, t)\right) d y>0$
$\int_{0}^{l}\left(\mu_{11_{t}}(y, t)-f(0, y, t)\right) d y<0, \int_{0}^{l}\left(\mu_{12_{t}}(y, t)-f(h, y, t)\right) d y>0, \mu_{22}(0, t)-\mu_{21}(0, t)$
$-\mu_{22}(h, t)+\mu_{21}(h, t) \geq 0, \mu_{31}^{\prime}(t)-\int_{D} f(x, y, t) d x d y>0, \mu_{32}^{\prime}(t)-\iint_{D} x f(x, y, t) d x d y>0$
$h>\frac{\mu_{32}^{\prime}(t)-\iint_{D} x f(x, y, t) d x d y,}{\mu_{31}^{\prime}(t)-\iint_{D}^{h} f(x, y, t) d x d y}>\frac{\int_{0}^{h}\left(\mu_{22}(x, t)-\mu_{21}(x, t)\right) d x}{\int_{0}^{h}\left(\mu_{22}(x, t)-\mu_{21}(x, t)\right) d x} \quad t \in[0, T]$.

Now find the solution of the system (6), (7):

$$
\begin{aligned}
& a_{1}(t)=\left(\left(\mu_{32}^{\prime}(t)-\iint_{D} x f(x, y, t) d x d y\right) \int_{0}^{h}\left(\mu_{22}(x, t)-\mu_{21}(x, t)\right) d x-\left(\mu_{31}^{\prime}(t)\right.\right. \\
& \left.\left.-\iint_{D} f(x, y, t) d x d y\right) \int_{0}^{h} x\left(\mu_{22}(x, t)-\mu_{21}(x, t)\right) d x\right) \Delta^{-1}(t), t \in[0, T] \\
& a_{2}(t)=\left(\left(\mu_{31}^{\prime}(t)-\iint_{D} f(x, y, t) d x d y\right) \int_{0}^{l}\left(h u_{x}(h, y, t)+\mu_{11}(y, t)-\mu_{12}(y, t)\right) d y\right. \\
& \left.-\left(\mu_{32}^{\prime}(t)-\iint_{D} x f(x, y, t) d x d y\right) \int_{0}^{l}\left(u_{x}(h, y, t)-u_{x}(0, y, t)\right) d y\right) \Delta^{-1}(t), t \in[0, T],
\end{aligned}
$$

where

$$
\begin{aligned}
& \Delta(t):=\int_{0}^{l}\left(h u_{x}(h, y, t)+\mu_{11}(y, t)-\mu_{12}(y, t)\right) d y \int_{0}^{h}\left(\mu_{22}(x, t)-\mu_{21}(x, t)\right) d x \\
& -\int_{0}^{l}\left(u_{x}(h, y, t)-u_{x}(0, y, t)\right) d y \int_{0}^{h} x\left(\mu_{22}(x, t)-\mu_{21}(x, t)\right) d x .
\end{aligned}
$$




\section{Investigation of The System (17)-(18)}

To establish the behavior of $\Delta(t)$ as $t \rightarrow 0$, we present it as

$$
\begin{aligned}
& \Delta(t)=\int_{0}^{l} u_{x}(h, y, t) d y \int_{0}^{h}(h-x)\left(\mu_{22}(x, t)-\mu_{21}(x, t)\right) d x+\int_{0}^{l} u_{x}(0, y, t) d y \int_{0}^{h} x\left(\mu_{22}(x, t)\right. \\
& \left.-\mu_{21}(x, t)\right) d x+\int_{0}^{l}\left(\mu_{11}(y, t)-\mu_{12}(y, t)\right) d y \int_{0}^{h}\left(\mu_{22}(x, t)-\mu_{21}(x, t)\right) d x
\end{aligned}
$$

Find $\int_{0}^{l} u_{x}(0, y, t) d y$ from $(10)$ and estimate the expressions

$$
\begin{aligned}
& \int_{0}^{h} G_{2}(0, t, \xi, 0) d \xi \int_{0}^{l} \varphi_{\xi}(\xi, \eta) d \eta \leq C_{1} \int_{0}^{h} G_{2}(0, t, \xi, 0) d \xi \\
& -\int_{0}^{t} G_{2}(0, t, 0, \tau) d \tau \int_{0}^{l}\left(\mu_{11_{\tau}}(\eta, \tau)-f(0, \eta, \tau)\right) d \eta \leq C_{2} \int_{0}^{t} G_{2}(0, t, 0, \tau) d \tau, \\
& \int_{0}^{t} G_{2}(0, t, h, \tau) d \tau \int_{0}^{t}\left(\mu_{12}(\eta, \tau)-f(h, \eta, \tau)\right) d \eta \leq C_{3} \int_{0}^{t} G_{2}(0, t, h, \tau) d \tau, \\
& \int_{0}^{t} G_{2}(0, t, 0, \tau) a_{2}(\tau)\left(\mu_{22}(0, \tau)-\mu_{21}(0, \tau)\right) d \tau \leq C_{4} \int_{0}^{t} G_{2}(0, t, 0, \tau) a_{2}(\tau) d \tau, \\
& \int_{0}^{t} G_{2}(0, t, h, \tau) a_{2}(\tau)\left(\mu_{22}(h, \tau)-\mu_{21}(h, \tau)\right) d \tau \leq C_{5} \int_{0}^{t} G_{2}(0, t, h, \tau) a_{2}(\tau) d \tau, \\
& -\int_{0}^{t} \int_{0}^{h} G_{2}(0, t, \xi, \tau) a_{2}(\tau) \mu_{21_{\xi}}(\xi, \tau) d \xi d \tau+\int_{0}^{h} \int_{0}^{h} G_{2}(0, t, \xi, \tau) a_{2}(\tau) \mu_{22_{\xi}}(\xi, \tau) d \xi d \tau \\
& \leq C_{6} \int_{0}^{h} \int_{0}^{t} G_{2}(0, t, \xi, \tau) a_{2}(\tau) d \xi d \tau, \\
& \int_{0}^{h} G_{2}(0, t, \xi, \tau) d \xi d \tau \int_{0} f_{\xi}(\xi, \eta, \tau) d \eta \leq C_{7} \int_{0}^{t} \int_{0}^{h} G_{2}(0, t, \xi, \tau) d \xi d \tau .
\end{aligned}
$$

Here and below $C_{i}, i=1,2,3, \ldots$, mean constants depending on given data. Denote $a_{i}(t):=$ $t^{\beta_{i}} b_{i}(t)$ and suppose that $\beta_{i} \geq 1, b_{i}(t)>0, t \in[0, T], i \in\{1,2\}$. Taking into account Green functions estimates [13], we get

$$
\int_{0}^{t} G_{2}(0, t, 0, \tau) d \tau \leq \int_{0}^{t}\left(\frac{C_{11}}{\sqrt{\theta_{1}(t)-\theta_{1}(\tau)}}+C_{12}\right) d \tau \leq \frac{C_{13} t^{\frac{1-\beta_{1}}{2}}}{\sqrt{\min _{[0, T]} b_{1}(t)}}+C_{12} T, G_{2}(0, t, h, \tau) \leq C_{14} .
$$


On the other hand,

$$
\int_{0}^{t} G_{2}(0, t, 0, \tau) d \tau \geq \int_{0}^{t} \frac{d \tau}{\sqrt{\pi\left(\theta_{1}(t)-\theta_{1}(\tau)\right)}} \geq \frac{C_{15} t^{\frac{1-\beta_{1}}{2}}}{\sqrt{\max _{[0, T]} b_{1}(t)}} .
$$

Hence, we obtain

$$
\begin{aligned}
& \frac{C_{16} t^{\frac{1-\beta_{1}}{2}}}{\sqrt{\max _{[0, T]} b_{1}(t)}} \leq \int_{0}^{l} u_{x}(0, y, t) d y \leq \frac{C_{17} t^{\frac{1-\beta_{1}}{2}}}{\sqrt{\min _{[0, T]} b_{1}(t)}}+\frac{C_{18}}{\sqrt{\min _{[0, T]} b_{1}(t)}} \int_{0}^{t} \frac{\tau^{\beta_{2}} b_{2}(\tau) d \tau}{\sqrt{t^{\beta+1}-\tau^{\beta+1}}} \\
& +C_{19} \int_{0}^{t} \tau^{\beta_{2}} b_{2}(\tau) d \tau .
\end{aligned}
$$

Transform the integral

$$
\int_{0}^{t} \frac{\tau^{\beta_{2}} b_{2}(\tau) d \tau}{\sqrt{t^{\beta+1}-\tau^{\beta+1}}} \leq t^{\beta_{2}-\beta_{1} / 2} \int_{0}^{t} \frac{b_{2}(\tau) d \tau}{\sqrt{t-\tau\left(\frac{\tau}{t}\right)^{\beta}}} \leq t^{\beta_{2}-\beta_{1} / 2} \int_{0}^{t} \frac{b_{2}(\tau) d \tau}{\sqrt{t-\tau}}
$$

Finally, we get

$$
\begin{aligned}
& \frac{C_{16} t^{\frac{1-\beta_{1}}{2}}}{\sqrt{\max _{[0, T]} b_{1}(t)}} \leq \int_{0}^{l} u_{x}(0, y, t) d y \leq \frac{C_{17} t^{\frac{1-\beta_{1}}{2}}}{\sqrt{\min _{[0, T]} b_{1}(t)}}+\frac{C_{20} t^{\beta_{2}-\beta_{1} / 2}}{\sqrt{\min _{[0, T]} b_{1}(t)}} \int_{0}^{t} \frac{b_{2}(\tau) d \tau}{\sqrt{t-\tau}} \\
& +C_{21} t^{\beta_{2}} \int_{0}^{t} b_{2}(\tau) d \tau \quad t \in(0, T] .
\end{aligned}
$$

Similarly we find

$$
\begin{aligned}
& \frac{C_{22} t^{\frac{1-\beta_{1}}{2}}}{\sqrt{\max _{[0, T]} b_{1}(t)}} \leq \int_{0}^{l} u_{x}(h, y, t) d y \leq \frac{C_{23} t^{\frac{1-\beta_{1}}{2}}}{\sqrt{\min _{[0, T]} b_{1}(t)}}+\frac{C_{24} t^{\beta_{2}-\beta_{1} / 2}}{\sqrt{\min _{[0, T]} b_{1}(t)}} \int_{0}^{t} \frac{b_{2}(\tau) d \tau}{\sqrt{t-\tau}} \\
& +C_{25} t^{\beta_{2}} \int_{0}^{t} b_{2}(\tau) d \tau \quad t \in(0, T] .
\end{aligned}
$$

It means that the following inequality is true:

$$
\begin{aligned}
& \frac{C_{26} t^{\frac{1-\beta_{1}}{2}}}{\sqrt{\max _{[0, T]} b_{1}(t)}} \leq \Delta(t) \leq \frac{C_{27} t^{\frac{1-\beta_{1}}{2}}}{\sqrt{\min _{[0, T]} b_{1}(t)}}+\frac{C_{28} t^{\beta_{2}-\beta_{1} / 2}}{\sqrt{\min _{[0, T]} b_{1}(t)}} \int_{0}^{t} \frac{b_{2}(\tau) d \tau}{\sqrt{t-\tau}}+C_{29} t^{\beta_{2}} \\
& \times \int_{0}^{t} b_{2}(\tau) d \tau, t \in(0, T] .
\end{aligned}
$$


Present (17) as

$$
\begin{aligned}
& a_{1}(t)=\Delta^{-1}(t) \int_{0}^{h}\left(\mu_{22}(x, t)-\mu_{21}(x, t)\right) d x\left(\mu_{31}^{\prime}(t)-\iint_{D} f(x, y, t) d x d y\right) \\
& \times\left(\frac{\mu_{32}^{\prime}(t)-\iint_{D} x f(x, y, t) d x d y,}{\mu_{31}^{\prime}(t)-\iint_{D} f(x, y, t) d x d y}-\frac{\int_{0}^{h} x\left(\mu_{22}(x, t)-\mu_{21}(x, t)\right) d x}{\int_{0}^{h}\left(\mu_{22}(x, t)-\mu_{21}(x, t)\right) d x}\right), \quad t \in(0, T] .
\end{aligned}
$$

Suppose that the following representations hold:

$$
\mu_{31}^{\prime}(t)-\iint_{D} f(x, y, t) d x d y \equiv \varkappa_{1}(t) t^{\frac{\beta_{1}+1}{2}}, \mu_{32}^{\prime}(t)-\iint_{D} x f(x, y, t) d x d y \equiv \varkappa_{2}(t) t^{\frac{\beta_{1}+1}{2}}
$$

where $\varkappa_{i}(t)>0, t \in[0, T], i \in\{1,2\}$. In virtue of assumptions (16) and (25), we obtain from (22):

$$
b_{1}(t) \leq C_{30} \sqrt{\max _{[0, T]} b_{1}(t)}
$$

or

$$
b_{1}(t) \leq B_{1}<\infty, \quad t \in[0, T],
$$

where constant $B_{1}$ is determined by given data.

Investigate the equation (18). Transform it as follows:

$$
\begin{aligned}
& a_{2}(t)=\left(\int_{0}^{l} u_{x}(h, y, t) d y\left(h \mu_{31}^{\prime}(t)-h \iint_{D} f(x, y, t) d x d y-\mu_{32}^{\prime}(t)+\iint_{D} x f(x, y, t) d x d y\right)\right. \\
& +\int_{0}^{l} u_{x}(0, y, t) d y\left(\mu_{32}^{\prime}(t)-\iint_{D} x f(x, y, t) d x d y\right)+\left(\mu_{31}^{\prime}(t)-\iint_{D} f(x, y, t) d x d y\right) \\
& \left.\times \int_{0}^{l}\left(\mu_{11}(y, t)-\mu_{12}(y, t)\right) d y\right) \Delta^{-1}(t), \quad t \in(0, T] .
\end{aligned}
$$

In addition to the assumptions from above, suppose that

$$
h \mu_{31}^{\prime}(t)-\mu_{32}^{\prime}(t)+\iint_{D}(x-h) f(x, y, t) d x d y>0, \quad t \in(0, T] .
$$

To estimate $a_{2}(t)$, note that from (15), (16) we have the inequality

$$
\int_{0}^{l} u_{x}(h, y, t) d y \geq \int_{0}^{l} u_{x}(0, y, t) d y
$$


Taking it and (20) into account, we obtain from (27)

$$
\begin{aligned}
& a_{2}(t) \leq\left(\mu_{31}^{\prime}(t)-\iint_{D} f(x, y, t) d x d y\right)\left(h \int_{0}^{l} u_{x}(h, y, t) d y+\int_{0}^{l}\left(\mu_{11}(y, t)-\mu_{12}(y, t)\right) d y\right) \\
& \times\left(\int_{0}^{l} u_{x}(0, y, t) d y \int_{0}^{h} x\left(\mu_{22}(x, t)-\mu_{21}(x, t)\right) d x\right)^{-1} .
\end{aligned}
$$

In virtue of (21), (22), (25), (26), we conclude

$$
\begin{aligned}
& a_{2}(t) \leq t^{\beta_{1}}\left(\frac{C_{31} t^{\frac{1-\beta_{1}}{2}}}{\sqrt{\min _{[0, T]} b_{1}(t)}}+\frac{C_{32} t^{\beta_{2}-\beta_{1} / 2}}{\sqrt{\min _{[0, T]} b_{1}(t)}} \int_{0}^{t} \frac{b_{2}(\tau) d \tau}{\sqrt{t-\tau}}+C_{33} t^{\beta_{2}} \int_{0}^{t} b_{2}(\tau) d \tau+C_{34}\right) \sqrt{\max _{[0, T]} b_{1}(t)} \\
& \leq t^{\frac{\beta_{1}+1}{2}}\left(\frac{C_{35}}{\sqrt{\min _{[0, T]} b_{1}(t)}}+\frac{C_{36} t^{\beta_{2}-1 / 2}}{\sqrt{\min _{[0, T]} b_{1}(t)}} \int_{0}^{t} \frac{b_{2}(\tau) d \tau}{\sqrt{t-\tau}}+C_{37} t^{\frac{\beta_{1}-1}{2}+\beta_{2}} \int_{0}^{t} b_{2}(\tau) d \tau+C_{38} t^{\frac{\beta_{1}-1}{2}}\right)
\end{aligned}
$$

or

$$
b_{2}(t) \leq \frac{C_{35} t^{\frac{\beta_{1}+1}{2}-\beta_{2}}}{\sqrt{\min _{[0, T]} b_{1}(t)}}+\frac{C_{36} t^{\frac{\beta_{1}}{2}}}{\sqrt{\min _{[0, T]} b_{1}(t)}} \int_{0}^{t} \frac{b_{2}(\tau) d \tau}{\sqrt{t-\tau}}+C_{37} t^{\beta_{1}} \int_{0}^{t} b_{2}(\tau) d \tau+C_{38} t^{\beta_{1}-\beta_{2}} .
$$

It is easy to see that the above inequality has sense if $\beta_{2}=\frac{\beta_{1}+1}{2}$. In this case we obtain

$$
b_{2}(t) \leq \frac{C_{35}}{\sqrt{\min _{[0, T]} b_{1}(t)}}+\frac{C_{36} t^{\frac{\beta_{1}}{2}}}{\sqrt{\min _{[0, T]} b_{1}(t)}} \int_{0}^{t} \frac{b_{2}(\tau) d \tau}{\sqrt{t-\tau}}+C_{37} t^{\beta_{1}} \int_{0}^{t} b_{2}(\tau) d \tau+C_{38} t^{\frac{\beta_{1}-1}{2}}, t \in[0, T] .
$$

Transform the integral

$$
\int_{0}^{t} b_{2}(\tau) d \tau=\int_{0}^{t} \frac{\sqrt{t-\tau} b_{2}(\tau) d \tau}{\sqrt{t-\tau}} \leq C_{39} \int_{0}^{t} \frac{b_{2}(\tau) d \tau}{\sqrt{t-\tau}}
$$

and reduce the inequality (29) to the form

$$
b_{2}(t) \leq \frac{C_{35}}{\sqrt{\min _{[0, T]} b_{1}(t)}}+\left(\frac{C_{40}}{\sqrt{\min _{[0, T]} b_{1}(t)}}+C_{41}\right) \int_{0}^{t} \frac{b_{2}(\tau) d \tau}{\sqrt{t-\tau}}+C_{42}, \quad t \in[0, T] .
$$

The inequality (30) is similar to the following one:

$$
v(t) \leq C_{43}+C_{44} \int_{0}^{t} \frac{v(\tau) d \tau}{\sqrt{t-\tau}}, \quad t \in[0, T] .
$$

To solve it, we find from (31)

$$
\int_{0}^{t} \frac{v(\sigma) d \sigma}{\sqrt{t-\sigma}} \leq C_{45}+C_{44} \int_{0}^{t} \frac{d \sigma}{\sqrt{t-\sigma}} \int_{0}^{\sigma} \frac{v(\tau) d \tau}{\sqrt{\sigma-\tau}} \leq C_{45}+C_{46} \int_{0}^{t} v(\tau) d \tau .
$$


Applying this estimate to (31) we obtain the Bellman-Gronwall inequality

$$
v(t) \leq C_{47}+C_{48} \int_{0}^{t} v(\tau) d \tau, \quad t \in[0, T]
$$

where $C_{47}, C_{48}$ are determined by $T, C_{43}, C_{44}$. The solution of (32) is given by

$$
v(t) \leq C_{47} e^{C_{48} t}, \quad t \in[0, T] .
$$

Hence, we have the following inequality for the solution of (30):

$$
b_{2}(t) \leq\left(\frac{C_{35}}{\sqrt{\min _{[0, T]} b_{1}(t)}}+C_{42}\right) \exp \left(\left(\frac{C_{40}}{\sqrt{\min _{[0, T]} b_{1}(t)}}+C_{41}\right) t\right), \quad t \in[0, T] .
$$

Return to the equation (24) and estimate $a_{1}(t)$ from below using (16), (23), (25):

$$
b_{1}(t) \geq \frac{C_{49} t^{\frac{1-\beta_{1}}{2}} \sqrt{\min _{[0, T]} b_{1}(t)}}{C_{27} t^{\frac{1-\beta_{1}}{2}}+C_{28} t^{\beta_{2}-\beta_{1} / 2} \int_{0}^{t} \frac{b_{2}(\tau) d \tau}{\sqrt{t-\tau}}+C_{29} t^{\beta_{2}} \sqrt{\min _{[0, T]} b_{1}(t)} \int_{0}^{t} b_{2}(\tau) d \tau} .
$$

In virtue of (26) and assumption $\beta_{2}=\frac{\beta_{1}+1}{2}$, we get

$$
b_{1}(t) \geq \frac{C_{49} \sqrt{\min _{[0, T]} b_{1}(t)}}{C_{27}+C_{28} \sqrt{t} \int_{0}^{t} \frac{b_{2}(\tau) d \tau}{\sqrt{t-\tau}}+C_{50} t^{\beta_{1}} \int_{0}^{t} b_{2}(\tau) d \tau} .
$$

Note that $C_{28} \sqrt{t} \int_{0}^{t} \frac{b_{2}(\tau) d \tau}{\sqrt{t-\tau}}+C_{50} t^{\beta_{1}} \int_{0}^{t} b_{2}(\tau) d \tau \rightarrow 0$ as $t \rightarrow 0$. Then $\exists T_{0} \in(0, T]:$

$$
C_{28} \sqrt{t} \int_{0}^{t} \frac{b_{2}(\tau) d \tau}{\sqrt{t-\tau}}+C_{50} t^{\beta_{1}} \int_{0}^{t} b_{2}(\tau) d \tau \leq C_{27}, \quad t \in\left[0, T_{0}\right] .
$$

One easily derives from here the estimate

$$
b_{1}(t) \geq B_{2}>0, \quad t \in\left[0, T_{0}\right] .
$$

Applying (37) to (34), we get

$$
b_{2}(t) \leq B_{3}<\infty, \quad t \in\left[0, T_{0}\right] .
$$

Here $B_{2}, B_{3}$ are known constants.

To establish the estimate of $a_{2}(t)$ from below, we find from $(27)$

$$
a_{2}(t) \geq \int_{0}^{l} u_{x}(0, y, t) d y\left(\mu_{32}^{\prime}(t)-\iint_{D} x f(x, y, t) d x d y\right) \Delta^{-1}(t) .
$$


After (38), (39) the estimate of $\Delta(t)$ from (23) becomes following:

$$
\Delta(t) \leq C_{51} t^{\frac{1-\beta_{1}}{2}}+C_{52}
$$

Similarly we get from (21):

$$
\int_{0}^{l} u_{x}(0, y, t) d y \geq C_{53} t^{\frac{1-\beta_{1}}{2}}
$$

Finally, we find

$$
b_{2}(t) \geq B_{4}>0, \quad t \in\left[0, T_{0}\right]
$$

where the constant $B_{4}$ is defined by given data.

4 Existence of Solution of the PRoblem (1)-(5)

Rewrite the equation (1) as

$$
u_{t}=b_{1}(t) t^{\beta_{1}} u_{x x}+b_{2}(t) t^{\beta_{2}} u_{y y}+f(x, y, t), \quad(x, y, t) \in Q_{T}
$$

and consider the inverse problem (40), (2)-(5) with unknowns $\left(b_{1}(t), b_{2}(t), u(x, y, t)\right)$.

Theorem. Suppose that in addition to (A1), (A2), the following assumptions hold:

(A3) $h \mu_{31}^{\prime}(t)-\mu_{32}^{\prime}(t)+\iint_{D}(x-h) f(x, y, t) d x d y>0, \mu_{31}^{\prime}(t)-\iint_{D} f(x, y, t) d x d y \equiv \varkappa_{1}(t) t^{\frac{\beta_{1}+1}{2}}$, $\mu_{32}^{\prime}(t)-\iint_{D} x f(x, y, t) d x d y \equiv \varkappa_{2}(t) t^{\frac{\beta_{1}+1}{2}}, \varkappa_{i}(t)>0, t \in[0, T], i \in\{1,2\} ;$

(A4) consistency conditions of zero order and conditions $\iint_{D} \varphi(x, y) d x d y=\mu_{31}(0)$, $\iint_{D} x \varphi(x, y) d x d y=\mu_{32}(0), \quad t \in(0, T]$.

If $\beta_{2}=\frac{\beta_{1}+1}{2}$, then there exists a solution $\left(b_{1}(t), b_{2}(t), u(x, y, t)\right)$ of the problem (40), (2)(5) defined for $(x, y) \in \bar{D}, t \in\left[0, T_{0}\right]$ such that $\left(b_{1}, b_{2}, u\right) \in\left(C\left(\left[0, T_{0}\right]\right)\right)^{2} \times C^{2,2,1}\left(D \times\left(0, T_{0}\right)\right) \cap$ $C^{1,0,0}\left(\bar{D} \times\left(0, T_{0}\right]\right), b_{i}(t)>0, t \in\left[0, T_{0}\right], i \in\{1,2\}$. The number $T_{0} \in(0, T]$ is determined by given data. 
Proof. Reduce the system (24), (27) to the form

$$
\begin{aligned}
& b_{1}(t)=t^{\frac{1-\beta_{1}}{2}} \varkappa_{1}(t) \int_{0}^{h}\left(\mu_{22}(x, t)-\mu_{21}(x, t)\right) d x\left(\frac{\mu_{32}^{\prime}(t)-\iint_{D} x f(x, y, t) d x d y,}{\mu_{31}^{\prime}(t)-\iint_{D} f(x, y, t) d x d y}\right. \\
& -\frac{\int_{0}^{h} x\left(\mu_{22}(x, t)-\mu_{21}(x, t)\right) d x}{h} \Delta_{0}^{-1}(t), \quad t \in(0, T], \\
& b_{2}(t)=\left(\left(h \varkappa_{22}(t)-\varkappa_{2}(t)\right) \int_{0}^{l} u_{x}(h, y, t) d y+\varkappa_{21}(x, t)\right) d x \\
& \left.+\varkappa_{1}(t) \int_{0}^{l}\left(\mu_{11}(y, t)-\mu_{12}(y, t)\right) d y\right) \Delta^{-1}(t), \quad t \in(0, T) d y .
\end{aligned}
$$

Evidently, the system (41), (42) is equivalent to the problem (40), (2)-(5). On the other hand, the estimates (26), (38)-(40) remain valid for its solution on $\left[0, T_{0}\right]$. Consider the system (41), (42) as an operator equation

$$
\omega=P \omega
$$

where $\left(b_{1}, b_{2}\right):=\omega,\left(P_{1}, P_{2}\right):=P$ and operators $P_{1}, P_{2}$ are defined by the right hand sides of the equations (41), (42), respectively. Consider the equation (43) in the domain $\mathcal{N}:=$ $\left\{\left(b_{1}, b_{2}\right) \in\left(C\left(\left[0, T_{0}\right]\right)\right)^{2}: B_{2} \leq b_{1} \leq B_{1}, B_{4} \leq b_{2} \leq B_{3}\right\}$ of the Banach space $\left(C\left(\left[0, T_{0}\right]\right)\right)^{2}$. In virtue of estimates (26), (38)-(40), the operator $P$ maps $\mathcal{N}$ onto itself. As it is shown in [7], [11], the operator is compact on $\mathcal{N}$. Then it follows from Schauder fixed-point theorem that the operator has at least one fixed point in $\mathcal{N}$. It means that the system (41), (42) has a solution $\left(b_{1}, b_{2}\right) \in\left(C\left(\left[0, T_{0}\right]\right)\right)^{2}$. Putting it in (8), we get a solution of the direct problem (40), (2)-(4) with appropriate smoothness [12].

\section{REFERENCES}

[1] Berestycki H., Busca H., Florent I. An inverse parabolic problem arising in finance. C. R. Acad. Sci. Paris. 2000, 331, 965-969. doi: 10.1016/S0764-4442(00)01749-3

[2] Caffarelli L., Friedman A. Continuity of the density of a gas flow in a porous medium. Trans. Amer. Math. Soc. 1979, 252, 99-113. doi: 10.2307/1998079

[3] DiBenedetto E. Degenerate parabolic equations. Springer, New York, 1993.

[4] Eldesbayev T. On an inverse problem for a degenerate hyperbolic equation of the second order. Proceedings of Academy of Sciences of KazSSR. Series of physics and mathematics. 1987, 3, 27-29. (in Russian)

[5] Gajiyev M. Inverse problem for a degenerate elliptic equation. Applications of functional analisys in methods of mathematical physics. 1987, 66-71. (in Russian)

[6] Ivanchov M., Saldina N. Inverse problem for a degenerate heat equation. Ukrainian Math. Journal. 2005, 57 (11), 1563-1570. doi: 10.1007/s11253-006-0032-6 (in Ukrainian) 
[7] Ivanchov M., Saldina N. Inverse problem for a parabolic equation with strong power degeneration. Ukrainian Math. Journal. 2006, 58 (11), 1487-1500. doi: 10.1007/s11253-006-0162-x (in Ukrainian)

[8] Ivanchov M., Saldina N. Inverse problem for strongly degenerate heat equation. J. Inv. Ill-Posed Problems. 2006, 14, 465-480. doi: 10.1515/156939406778247598

[9] Ivanchov M., Vlasov V. Inverse problem for a weakly degenerate two-dimensional heat equation. Visnyk Lviv. Univer. Ser. Mech.-Math. 2009, 70, 91-102. (in Ukrainian)

[10] Vlasov V. Inverse problem for a weakly degenerate two-dimensional anisotropic parabolic equation. Bukovynsky Math. Journal. 2017, 5 (1-2), 37-48. (in Ukrainian)

[11] Ivanchov M., Vlasov V. Inverse problem for a two-dimensional strongly degenerate heat equation. Electronic Journal of Differential Equations. 2018, 77, 1-17.

[12] Ladyzhenskaya O., Solonnikov V., Uraltseva N. Linear and quasilinear equations of the parabolic type. Nauka, Moscow, 1967.

[13] Ivanchov M. Inverse problems for equations of parabolic type. VNTL Publishers, Lviv, 2003.

Received 01.07.2019

Микола Іванчов, Віталій Власов Обернена задача для двовимірного рівняння теплопровідності зі сильним виродженням. Випадок інтегральних умов перевизначення. // Буковинський матем. журнал - 2019. - Т.7, №1. - С. 32-47.

Розглядається існування класичного розв'язку оберненої задачі знаходження двох залежних від часу коефіцієнтів у двовимірному рівнянні теплопровідності. Припускаємо, що невідомі коефіцієнти зникають у початковий момент часу як степенева функція із показником, більшим 1. Існування доводиться з допомогою теореми Шаудера про нерухому точку. 